\title{
Índice de masa corporal y niveles séricos de lípidos
}

Pedro Javier Navarrete Mejía ${ }^{1,2}$, Manuel Jesús Loayza Alarico ${ }^{1,2}$, Juan Carlos Velasco Guerrero ${ }^{1,2}$, Zoel Aníbal Huatuco Collantes ${ }^{1,3}$, Ruth Angélica Abregú Meza ${ }^{4}$

\section{RESUMEN}

Objetivo: Identificar la asociación entre el índice de masa corporal (IMC) y los niveles séricos de lípidos en personas adultas.

Material y Métodos: Estudio de tipo observacional, transversal y retrospectivo. Diseño de investigación no experimental. La población estuvo conformada por atendidas en establecimientos de salud privados de la ciudad de Lima Metropolitana. Las evaluaciones del IMC y pruebas laboratoriales para determinar concentraciones séricas de lípidos se realizaron entre octubre del 2014 y octubre del 2015. Se determinó la asociación entre Índice de masa corporal y niveles séricos de lípidos mediante la prueba de $\mathrm{Chi}^{2}$. Se excluyó personas con comorbilidades que pueden alterar los niveles séricos de lípidos.

Resultados: El $39.7 \%$ y $60.3 \%$ de las personas evaluadas fueron de sexo masculino y femenino respectivamente. La edad promedio fue de 34,2 años. La población presentó en un 40.7\% (1227/3016) sobrepeso u obesidad. Los resultados muestran mayor sobrepeso u obesidad en los pacientes del sexo masculino que femenino (54.6\% y $33 \%$ respectivamente). El $19.7 \%$ (594/3016) de las personas evaluadas presentó niveles altos de triglicéridos, el $27.9 \%(841 / 3016)$ presentó niveles altos de colesterol y el 38,8\% (1146/3016) presentó bajos niveles de cHDL. Los niveles de cLDL y cVLDL fueron similares en ambos sexos.

Conclusiones: La investigación determinó asociación estadísticamente significativa entre el IMC y los triglicéridos $(p<0.05)$, colesterol $(p<0.05)$ y $c H D L ~(p<0.05)$.

Palabras clave: índice de masa corporal, colesterol sérico, triglicéridos sérico, HDL.

\section{Body mass index and serum lipid levels}

\section{ABSTRACT}

Objective: To identify the association between the body mass index (BMI) and serum lipid levels in adult people.

Material and Methods: Observational, transversal and retrospective study. Non experimental investigation design. The population was conformed for people treated in private health centers in Metropolitan Lima. The evaluations of the BMI and the laboratorial tests to know the seric concentration of lipids were taken between October 2014 and October 2015 . It was determined the association between the BMI and the seric lipid levels using the Chi2 test. People with comorbidity that could modify the seric levels of lipids were excluded.

Results: $39.7 \%$ of people studied were male and $60.3 \%$ were female. The average age was 34.2 years old. $40.7 \%$ (1227/3016) of population were obese and overweight. The results show a higher level of obesity or overweight in male people over female $(54.6 \%$ and $33 \%$ respectively). $19.7 \%$ (594/3016) of the tested people presented high triglycerides seric levels. 27.9\% (841/3016) presented high cholesterol levels and 38.8\% (1146/3016) presented low cHDL levels. The cLDL levels and cVLDL levels were similar in both groups (male and female).

Conclusions: The investigation determined the significant statistical association between the BMI and triglycerides ( $p<$ $0.05)$, cholesterol $(p<0.05)$ and $\operatorname{cHDL}(p<0.05)$.

Key words: body mass index, seric cholesterol, seric triglycerides, HDL.

1. Centro de Investigación de Salud Pública. Instituto de Investigación. Facultad de Medicina Humana. Universidad de San Martín de Porres.

2. Doctor en Salud Pública.

3. Doctor en Medicina.

4. Licenciada en Nutrición. Maestra en Bioquímica y Nutrición. 
Pedro Javier Navarrete Mejía, Manuel Jesús Loayza Alarico, Juan Carlos Velasco Guerrero, Zoel Aníbal Huatuco Collantes, Ruth Angélica Abregú Meza

\section{INTRODUCCIÓN}

La obesidad es la principal enfermedad crónica no transmisible a la cual los organismos internacionales como la Organización Mundial de la Salud (OMS) han puesto atención, no olvidemos que la obesidad tiene una alta prevalencia y está asociada a un número importante de otras enfermedades.

La obesidad y el sobrepeso en la población peruana han venido de menos a más progresivamente en las últimas décadas, este es un problema no solo de nuestra población sino también de otras sociedades desarrolladas; la OMS califica el problema como una epidemia. La obesidad es el primer eslabón de una cadena que conduce a la muerte por enfermedad cardiovascular a través de la ateroesclerosis (1).

Los países están haciendo grandes esfuerzos para combatir la obesidad y el sobrepeso, en Europa se destina en promedio el $2.8 \%$ del presupuesto en salud (2), en los EE. UU el gastó para esta epidemia puede llegar a cifras superiores a los 150 mil millones de dólares (3), y en el caso peruano se estima que los costos directos así como las pérdidas económicas como consecuencia de la enfermedad y el impacto sobre la calidad de vida de quienes la padecen pueda llegar a costar 2 mil millones de soles anuales.

La OMS define el índice de masa corporal (IMC) como un indicador simple de la relación entre el peso y la talla que puede utilizarse para identificar el sobrepeso y la obesidad en los adultos. Un IMC igual o superior a 25 determina sobrepeso y un IMC igual o superior a 30 determina obesidad.

Existen parámetros fisiológicos, bioquímicos o moleculares (biomarcadores) asociados a la severidad de determinadas enfermedades, su medición permite conocer el estado de salud de un individuo (4). Los lípidos séricos son ejemplos de biomarcadores, los niveles en suero son de indiscutible valor diagnóstico y pronóstico para enfermedades vasculares de tipo aterosclerótico (5).
La investigación busca conocer para la población de estudio la relación entre IMC y biomarcadores (lípidos séricos).

\section{MATERIAL Y MÉTODOS}

Estudio de tipo observacional, transversal y retrospectivo. Diseño de investigación no experimental.

La población estuvo conformada por 3016 personas atendidas en establecimientos de salud privados de la ciudad de Lima Metropolitana. Las unidades de análisis fueron personas que en forma voluntaria acudieron para controles nutricionales y evaluación de riesgo cardiovascular.

La determinación del IMC fue realizada por profesionales en nutrición y dietética, las evaluaciones laboratoriales se realizaron en ayunas y por profesionales acreditados para el desarrollo de pruebas bioquímicas.

Las evaluaciones del IMC y pruebas laboratoriales para determinar concentraciones séricas de lípidos se realizaron entre octubre del 2014 y octubre del 2015. Se recolectó información sobre las variables de estudio sexo, peso, talla, IMC y concentración sérica de lípidos. Los datos fueron recolectados en una ficha elaborada para investigación, la fuente de información fueron las historias clínicas.

Los datos fueron procesados usando el paquete estadístico SPSS v23. Se determinó la asociación entre IMC y niveles séricos de lípidos mediante la prueba de $\mathrm{Chi}^{2}$.

\section{RESULTADOS}

Se evaluó el IMC y niveles séricos de lípidos (colesterol, triglicéridos, HDL, LDL y VLDL) de 3016 personas. El $39.7 \%$ y $60.3 \%$ de las personas evaluadas fueron de sexo masculino y femenino respectivamente. La edad promedio fue de 34,2 años (Tabla 1).

Tabla 1. Población en estudio

\begin{tabular}{|cccc|}
\hline Variable & Masculino & Femenino & Total \\
\hline Edad * & $32.6+/-5.6$ años & $36.1+/-8.0$ años & $34.2+/-7.8$ años \\
\hline Sexo & $39.7 \%$ & $60.3 \%$ \\
\hline
\end{tabular}

${ }^{*}$ Rango edades $=$ Max-Min $=47-18=29$ años 
Los IMC y valores del perfil lipídico se expresan en la tabla 2.

Tabla 2. Medias y desviación de variables estudiadas

\begin{tabular}{|lcc|}
\hline Índice de Masa Corporal & Masculino & Femenino \\
\hline Muy bajo peso $(<16.9)$ & $15.1+/-0.4$ & $14.9+/-0.6$ \\
\hline Bajo peso $(17.0-18.4)$ & $17.6+/-0.7$ & $17.4+/-0.2$ \\
\hline Normal $(18.5-24.9)$ & $22.2+/-2.2$ & $23.0+/-1.1$ \\
\hline Sobrepeso $(25.0-29.9)$ & $28.1+/-1.2$ & $28.9+/-0.4$ \\
\hline Obesidad $(30.0-34.9)$ & $33.0+/-1.1$ & $32.2+/-2.0$ \\
\hline Obesidad marcada (35.0 -39.9) & $38.0+/-1.1$ & $38.0+/-1.0$ \\
\hline Obesidad mórbida $(>40.0)$ & $42.6+/-2.0$ & $43.0+/-2.6$ \\
\hline Nivel Sérico & & \\
\hline Triglicéridos $>150 \mathrm{mg} / \mathrm{dl}$ & $197.2+/-38.0$ & $188.0+/-27.2$ \\
\hline Colesterol $>200 \mathrm{mg} / \mathrm{dl}$ & $261.6+/-51.3$ & $250.2+/-39.1$ \\
\hline $\mathrm{CHDL}<40 \mathrm{mg} / \mathrm{dl}$ & $28.2+/-9.3$ & $27.0+/-9.1$ \\
\hline $\mathrm{CLDL}>100 \mathrm{mg} / \mathrm{dl}$ & $135.2+/-16.2$ & $126.3+/-10.1$ \\
\hline $\mathrm{CVLDL}>40 \mathrm{mg} / \mathrm{dl}$ & $46.2+/-3.6$ & $49.2+/-2.2$ \\
\hline
\end{tabular}

La población presentó en un 40.7\% (1227/3016) sobrepeso u obesidad. Los resultados muestran mayor sobrepeso, obesidad y obesidad marcada en los pacientes del sexo masculino que femenino
(40.9\%, $8.4 \%$ y $4.9 \%$ respectivamente). En ambos sexos se encontró la misma prevalencia de obesidad mórbida $(0.4 \%)$ (Tabla 3$)$.

Tabla 3. Índice de masa corporal según sexo

\begin{tabular}{|lrrr|}
\hline Índice de Masa Corporal & Masculino & Femenino & Total \\
\hline Muy bajo peso $(<16.9)$ & $0,4 \%$ & $1,1 \%$ & $0,6 \%$ \\
\hline Bajo peso $(17.0-18.4)$ & $0,4 \%$ & $1,2 \%$ & $0,7 \%$ \\
\hline Normal $(18.5-24.9)$ & $44,6 \%$ & $64,7 \%$ & $58,0 \%$ \\
\hline Sobrepeso $(25.0-29.9)$ & $40,9 \%$ & $26,7 \%$ & $32,7 \%$ \\
\hline Obesidad (30.0-34.9) & $8,4 \%$ & $4,4 \%$ & $5,9 \%$ \\
\hline Obesidad marcada (35.0-39.9) & $4,9 \%$ & $1,5 \%$ & $1,7 \%$ \\
\hline Obesidad mórbida (> 40.0) & $0,4 \%$ & $0,4 \%$ & $0,4 \%$ \\
\hline
\end{tabular}

El 19.7\% (594/3016) de las personas evaluadas presentó niveles altos de triglicéridos, el $27.9 \%$ (841/3016) niveles altos de colesterol y el $38,8 \%$ (1146/3016) bajos niveles de cHDL. Los pacientes del sexo masculino tienen mayor prevalencia de hipertrigliceridemia, hipercolesterolemia y niveles séricos más bajos de $\mathrm{CHDL}$ en relación al sexo femenino (triglicéridos: $21 \%$ y $9 \%$; Colesterol: $41,2 \%$ y 20\%; cHDL: $52.5 \%$ y $13.2 \%$ ). Los niveles de cLDL y cVLDL fueron similares en ambos sexos (Tabla 4). 
Tabla 4. Perfil lipídico según sexo

\begin{tabular}{|lcrc|}
\hline \multicolumn{1}{|c}{ Nivel Sérico } & Masculino & Femenino & Total \\
\hline Triglicéridos $>150 \mathrm{mg} / \mathrm{dl}$ & $21 \%$ & $9 \%$ & $19.7 \%$ \\
\hline Colesterol $>200 \mathrm{mg} / \mathrm{dl}$ & $41.2 \%$ & $20 \%$ & $27.9 \%$ \\
\hline $\mathrm{CHDL}<40 \mathrm{mg} / \mathrm{dl}$ & $52.5 \%$ & $13.2 \%$ & $38.8 \%$ \\
\hline $\mathrm{CLDL}>100 \mathrm{mg} / \mathrm{dl}$ & $13.9 \%$ & $13.9 \%$ & $13.9 \%$ \\
\hline $\mathrm{cVLDL}>40 \mathrm{mg} / \mathrm{dl}$ & $11.4 \%$ & $11.4 \%$ & $11.4 \%$ \\
\hline
\end{tabular}

La investigación permitió determinar asociación estadísticamente significativa entre el IMC y los niveles de triglicéridos $(\mathrm{p}<0.05)$, IMC y los niveles de colesterol $(p<0.05)$ e IMC y los niveles de cHDL $(p<0.000)$ (Tabla 5).

Tabla 5. Índice de masa corporal y lípidos séricos

\begin{tabular}{|c|c|c|c|}
\hline \multirow[t]{2}{*}{ IMC $\left(\mathrm{kg} / \mathrm{m}^{2}\right)$} & \multicolumn{2}{|c|}{ Lípido } & \multirow[t]{2}{*}{$\mathrm{p}$ valor } \\
\hline & Triglicéridos $<=150 \mathrm{mg} / \mathrm{dl}$ & Triglicéridos $>150 \mathrm{mg} / \mathrm{dl}$ & \\
\hline Bajo peso (< $16.9-18.4)$ & $0.6 \%$ & $0.7 \%$ & \multirow{3}{*}{0.021} \\
\hline Normal (18.5-24.9) & $55.4 \%$ & $2.6 \%$ & \\
\hline \multirow[t]{2}{*}{ Sobre peso/Obesidad $(25->40)$} & $24.3 \%$ & $16.4 \%$ & \\
\hline & Colesterol < = $200 \mathrm{mg} / \mathrm{dl}$ & Colesterol > 200 mg/dl & \\
\hline Bajo peso $(<16.9-18.4)$ & $1.2 \%$ & $0.1 \%$ & \multirow{3}{*}{0.013} \\
\hline Normal (18.5-24.9) & $55.9 \%$ & $2.1 \%$ & \\
\hline \multirow{2}{*}{ Sobre peso/Obesidad (25->40) } & $15 \%$ & $25.7 \%$ & \\
\hline & $\mathrm{cHDL}<40 \mathrm{mg} / \mathrm{dl}$ & $\mathrm{cHDL}>=40 \mathrm{mg} / \mathrm{dl}$ & \\
\hline Bajo peso $(<16.9-18.4)$ & $0.1 \%$ & $1.2 \%$ & \multirow{3}{*}{0.000} \\
\hline Normal (18.5-24.9) & $4.4 \%$ & $53.6 \%$ & \\
\hline \multirow[t]{2}{*}{ Sobre peso/Obesidad (25->40) } & $34.3 \%$ & $6.4 \%$ & \\
\hline & $\mathrm{cLDL}<=100 \mathrm{mg} / \mathrm{dl}$ & $\mathrm{cLDL}>100 \mathrm{mg} / \mathrm{dl}$ & \\
\hline Bajo peso $(<16.9-18.4)$ & $1.2 \%$ & $0.1 \%$ & \multirow{3}{*}{0.07} \\
\hline Normal (18.5-24.9) & $53.9 \%$ & $4.1 \%$ & \\
\hline \multirow[t]{2}{*}{ Sobre peso/Obesidad (25->40) } & $31 \%$ & $9.7 \%$ & \\
\hline & $\mathrm{cVLDL}<=40 \mathrm{mg} / \mathrm{dl}$ & $\mathrm{cVLDL}>40 \mathrm{mg} / \mathrm{dl}$ & \\
\hline Bajo peso $(<16.9-18.4)$ & $0.3 \%$ & $1.0 \%$ & \multirow{3}{*}{0.051} \\
\hline Normal (18.5-24.9) & $53 \%$ & $5.0 \%$ & \\
\hline Sobre peso/Obesidad (25 ->40) & $35.3 \%$ & $5.4 \%$ & \\
\hline
\end{tabular}




\section{DISCUSIÓN}

Los malos hábitos, los cambios en la dieta y la poca actividad física traen como consecuencia el incremento de peso y la obesidad en la población, el exceso de peso se relaciona con la aparición de enfermedades cardíacas, metabólicas, renales, desórdenes del sueño, cáncer y problemas mentales (6).

Según el estudio Cardiovascular Risk Factor Multiple Evaluation in Latin America, investigación que evaluó la prevalencia de factores de riesgos cardiovasculares en siete ciudades de Latinoamérica, reportó una prevalencia de obesidad del $18 \%$ al $31 \%$ de la población (7). En la actualidad países como México y Colombia presentan la mayor prevalencia de sobrepeso y obesidad (México: Hombres 66,8\% y Mujeres 71,4\%; Colombia: Hombres $52.7 \%$ y Mujeres $57 \%$ ) (8). La presente investigación - para el grupo en estudio - presenta cifras inferiores para el caso de los hombres $(39.7 \%)$ y cercanas en el caso de las mujeres $(60.3 \%)$.

Entre los principales factores de riesgo con un rol fundamental en el desarrollo de enfermedades crónicas, se encuentra presente la obesidad. Las enfermedades cardiovasculares son causa de muerte en la población peruana, es la enfermedad isquémica coronaria entre las cardiopatías, la principal causa de mortalidad. En Latinoamérica, los factores de riesgo cardiovascular presentan una prevalencia similar a la de los países desarrollados, pero con menos recursos para su tratamiento y prevención $(7,9,10)$.

La alta concentración de lípidos séricos condiciona enfermedades como la arteriosclerosis, las grasas se acumulan y depositan en las paredes de las arterias, disminuyendo su elasticidad y reduciendo su diámetro $(11,12,13)$.

En el estudio se determinó que el $19.7 \%$ de la población presenta concentraciones séricas de triglicéridos superiores a $150 \mathrm{mg} / \mathrm{dl}$, estamos frente a un grupo poblacional en riesgo, recordemos que las altas concentraciones están asociadas a infarto de miocardio e ictus isquémico; por otro lado es necesario no olvidar que los triglicéridos altos también afectan los riñones (disminución de la función renal), el páncreas (insuficiencia pancreática) y el hígado (hígado graso). El grupo de estudio con mayor prevalencia de hipertrigliceridemia fue el grupo con obesidad/ sobrepeso (16.4\%). Es importante mencionar que el
3.3\% de los pacientes con niveles altos de triglicéridos presentaron un IMC normal o bajo.

El nivel alto de colesterol es una de las enfermedades silenciosas (hipercolesterolemia), situación por la cual es importante la prevención en búsqueda de evitar las complicaciones $(2,7,14)$. La obesidad/sobrepeso son de por si problemas serios para la salud de los individuos, que sumado a niveles elevados de colesterol generan un pronóstico nada alentador. La bibliografía existente es extensa sobre los riesgos coronarios como consecuencia de hipercolesterolemia $(15,16)$. El $25.7 \%$ de la población estudiada presenta niveles de colesterol superiores a los normales (>200 mg/dl), la investigación también refleja que existe población con niveles altos de colesterol y no necesariamente pertenece al grupo de personas con obesidad/sobrepeso (2.3\%), y en el otro extremo existe un $15 \%$ de personas con niveles normales de colesterol sérico a pesar de pertenecer al grupo de riesgo.

El cHDL es de interpretación inversa al triglicérido y colesterol con respecto a su concentración sérica, niveles altos garantizan menor riesgo y concentraciones bajas $(<40 \mathrm{mg} / \mathrm{dl})$ mayor riesgo, principalmente para enfermedades cardiacas. La población estudiada presentó en un $38.8 \%$ concentraciones séricas por debajo del límite teórico de protección ante riesgo coronario. El 34.3\% de los investigados con concentraciones séricas de riesgo coronario pertenecen al grupo de individuos con obesidad/sobrepeso, pero también se determinó que un importante $4.5 \%$ de la población con concentraciones bajas de cHDL eran normales o con bajo peso desde el punto de vista del IMC.

El CLDL es una combinación de grasa y proteína que entrega el colesterol y otras grasas a las células del cuerpo humano. Concentraciones muy altas de cLDL (>100 mg/dl) coadyuvan a la formación de placas en las paredes de las arterias, se incrementa el riesgo de un infarto o ACV $(17,18)$. El $13.9 \%$ de la población estudiada presentó niveles superiores de CLDL, al límite superior esperado sin riesgo coronario. El 9.7\% de los investigados que presentó cLDL elevado tenían obesidad/sobrepeso, pero el $4.2 \%$ estaban en un IMC de normalidad o bajo peso según IMC. Situación similar con el cVLDL, el $11.4 \%$ de la población presenta niveles altos $(40 \mathrm{mg} / \mathrm{dl})$, riesgo presente para enfermedades coronarias.

La investigación permite afianzar el conocimiento sobre la creencia que los individuos con obesidad/sobrepeso son los que tiene problemas con 
Pedro Javier Navarrete Mejía, Manuel Jesús Loayza Alarico, Juan Carlos Velasco Guerrero, Zoel Aníbal Huatuco Collantes, Ruth Angélica Abregú Meza

las concentraciones séricas de lípidos, verdad a medias, pues el $3.1 \%$ de las hipertrigliceridemias, el $2.2 \%$ de las hipercolesterolemias, el $4.5 \%$ del $\mathrm{cHDL}$ bajo, el $4.1 \%$ del cLDL y el $6 \%$ de cVLDL alto se presentaron en pacientes con IMC normal o bajo.

En conclusión, el IMC puede ser un parámetro a tener en cuenta para proyectar teóricamente posibles alteraciones séricas en los lípidos, pero no es mandatorio, la fisiología y tal vez factores genéticos no permiten estandarizar este criterio, si bien es cierto - en la presente investigación - el triglicérido, colesterol y cHDL son estadísticamente significativos en su relación con el IMC, se hace necesario siempre evaluaciones de riesgo coronario (u otras alteraciones asociadas a perfil lipídico) en la personas independientemente de su IMC.

\section{REFERENCIAS BIBLIOGRÁFICAS}

1. Rosas A, Lama G, Llanos F. Prevalencia de Obesidad e Hipercolesterolemia en trabajadores de una Institución estatal de Lima - Perú. 2002. Rev. peru. med. exp. salud publica;19(2):87-92.

2. Muller-Riemenschneider F, Reinhold T, Nocon M, Willich SN. Long-term effectiveness of interventions promoting physical activity: a systematic review. 2008. Prev Med. 47(4):354-68.

3. Finkelstein EA, Trogdon JG, Cohen JW, Dietz W. Annual medical spending attributable to obesity: payer-and servicespecific estimates. 2009. Health Aff (Millwood). 28(5): 822-31.

4. Cohen S, Oates JA, Spilker B, Zegerc SL. Establishing the research infrastructure to develop and classify clinical biomarkers. In: Dowing GJ, editor. Biomarkers and surrogate endpoint: Clinical research and applications. 2000. New York: Elsevier p. 307-14.

5. Brunzell JD, Davidson M, Furberg CD, Goldberg RB. Lipoprotein management in patients with cardiometabolic risk: consensus statement from the American Diabetes Association and the American College of Cardiology Foundation. 2008. Diabetes Care. 31:811-22.

6. Canoy D, Cairns BJ, Balkwill A, Wright FL, Green J, Reeves G, et al. Body mass index and incident coronary heart disease in women: a population-based prospective study. 2013. BMC Med. 11(1):87.

7. Schargrodsky $H$, Hernández-Hernández R, Champagne BM, Silva H, Vinueza R, Silva Ayçaguer LC, et al. CARMELA: Assessment of Cardiovascular Risk in Seven Latin American Cities. 2008. Am J Med. 121(1):58-65

8. Ng M, Fleming T, Robinson M, Thomson B, Graetz N, Margono $C$, et al. Global, regional, and national prevalence of overweight and obesity in children and adults during 1980-2013: a systematic analysis for the Global Burden of Disease Study 2013. 2014. The Lancet. 384(9945):766-81.

9. Querales M, Sánchez C. Dislipidemias en un grupo de adultos aparentemente sanos. 2013. Salus; 17(1): 7-11.

10. Bojorquez L, Saucedo T, Juárez F. Medio ambiente social y conductas alimentarias de riesgo: una exploración en mujeres adolescentes en México. 2012. Cad Saude Pública; 29(1): 29-39.

11. Bello B, Sánchez G, Ferreira A, Báez E. Síndrome Metabólico: un problema de salud con múltiples definiciones. 2012. Rev. Médica electron; 34(2): 199-213
12. Ramírez R. El sedentarismo es un factor predictor de hipertrigliceridemia, obesidad central y sobrepeso. 2012. Rev. colomb. cardiol; 19(2): 75-79

13. González L, Estrada-L. Exceso de peso, aspectos económicos, políticos y sociales en el mundo: Un análisis ecológico. 2011. Cad. Saúde Pública vol.27 No.9.

14. Delgado Jo, Cardona L, Rodriguez M, Toro A. Perfil clínico y epidemiológico de los pacientes de un programa de riesgo cardiovascular.2011.Med. U.P.B; 30(2): 132-140.

15. Málaga G, Zevallos C, Lazo M. Elevada frecuencia de dislipidemias y glucemia basal alterada en una población Peruana de altura. 2010. Rev Peru Med Exp Salud Pública; 27(4): 557-561.

16. 16 Navarrete C, Cartes R, Carrasco C. Dislipidemias en comunidades pehuenches de Alto Biobio chileno. 2011. Medisan; 17(1): 68-77.

17. Costa B, Horta N, Souza G. Índice de masa corporal presenta buena correlación con el proaterosclerótico en niños y adolescentes. 2009. Arq Bras Cardiol; 93(3): 261-267.

18. Bermúdez J, Velásquez C. Perfil de ácidos grasos libres (AGL) en suero de jóvenes colombianos con obesidad y síndrome metabólico.2014. Arch. latinoam. nutr; 64(4): 248-257.

\section{Fuentes de Financiamiento}

Este artículo ha sido financiado por los autores.

\section{Coflictos de interés}

Los autores declaran no presentar ningún conflicto de interés.

\section{Correspondencia:}

Pedro Javier Navarrete Mejía

Dirección: Av. Alameda del Corregidor No 1531Urb Los Sirius III

Etapa La Molina. Lima. Perú

Teléfono: 998998844

Correo: pnavarretem@usmp.pe 